\title{
Theory and Design of a Class of $M$-Channel IIR Cosine-Modulated Filter Banks
}

\author{
J. S. Mao, S. C. Chan, and K. L. Ho
}

\begin{abstract}
This letter proposes a method for designing a class of $M$-channel, causal, stable, perfect reconstruction (PR) IIR cosine-modulated filter banks (CMFB). The proposed CMFB has the same denominator for all its polyphase components in the prototype filter. Therefore, the PR condition is considerably simplified, and it is relatively simple to satisfy the PR and the casual-stable requirements of the IIR CMFB. Design examples show that the proposed IIR CMFB has sharper cutoff, higher stopband attenuation, and passband flatness than its FIR counterparts, especially when the system delay is small.
\end{abstract}

Index Terms-Causal stable, cosine-modulated, filter banks, IIR, perfect reconstruction.

\section{INTRODUCTION}

$\mathbf{P}$ ERFECT reconstruction (PR) filter banks have important applications in signal analysis, coding, and the design of wavelet bases [1]. Fig. 1 shows the block diagram of an $M$-channel maximally decimated filter bank. The FIR cosine-modulated filter banks (CMFB) [2] or the extended lapped transforms [8] have emerged as very good tradeoff between filter quality, implementation, and design complexities. Recently, the theory and design of the more general arbitrary length, biorthogonal CMFB has also been studied [3], [4]. One application of such biorthogonal CMFB is to realize filterbank with low system delay. Although IIR filter banks can potentially offer low system delay and high stopband attenuation, their design is very involved. They are either limited to the two-channel case [5] or suffer from stability problems [7]. In fact, the difficulty in designing such IIR filter banks is to satisfy the complicated PR condition and the causal-stable requirement of the filters. In this paper, a novel method for designing a class of $M$-channel, causal-stable PR IIR CMFB is proposed. The proposed IIR CMFB has the same denominator for all the polyphase components of its prototype filter. Therefore, the PR condition is considerably simplified, and it is simpler to satisfy the PR and the casual stable requirements. Design examples show that the proposed IIR CMFB has sharper cutoff and higher stopband attenuation and passband flatness than its FIR counterparts, especially when the system delay is small. The layout of the letter is as follows. Section II is devoted to the theory of the proposed $M$-channel IIR CMFB. Their design procedure and several examples will be given in

\footnotetext{
Manuscript received September 28, 1999. The associate editor coordinating the review of this manuscript and approving it for publication was Prof. R. Shenoy.

The authors are with the Department of Electrical and Electronic Engineering, The University of Hong Kong, Kowloon, Hong Kong (e-mail: klho@eee.hku.hk).

Publisher Item Identifier S 1070-9908(00)00923-8.
}

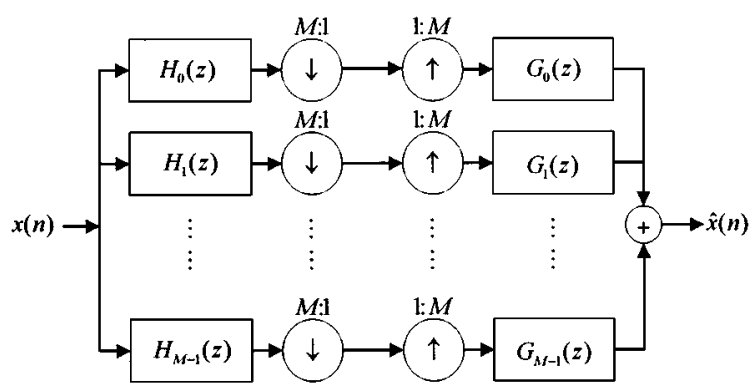

Fig. 1. $M$-channel maximally decimated filter banks.

Section III. Finally, the results are summarized in Section IV, the conclusion.

\section{PRoposed $M$-ChANNEL PR IIR CMFB}

The analysis filters $f_{k}(n)$ and the synthesis filters $g_{k}(n)$ in a CMFB are obtained by modulating a prototype filter $h(n)$ as follows:

$$
\begin{gathered}
f_{k}(n)=h(n) c_{k, n}, \quad g_{k}(n)=h(n) \bar{c}_{k, n}, \\
k=0,1, \cdots, M-1
\end{gathered}
$$

where $M$ is the number of channels. The modulation we shall be using is the extended lapped transform (ELT) [8] given by

$$
c_{k, n}=\sqrt{\frac{2}{M}} \cos \left[(2 k+1) \frac{\pi}{2 M}\left(n+\frac{M+1}{2}\right)\right]
$$

and $\bar{c}_{k, n}$ is the time reverse of $c_{k, n}$. The PR condition for the biorthogonal CMFB is given by [3], [4]

$$
\begin{aligned}
& H_{k}(z) H_{2 M-k-1}(z)+H_{M+k}(z) H_{M-k-1}(z)=\beta \cdot z^{-n_{k}}, \\
& \quad k=0,1, \cdots,(M / 2)-1
\end{aligned}
$$

where $H_{k}(z)$ is the type-I polyphase component of the prototype filter $H(z)=\sum_{k=0}^{2 M-1} z^{-k} H_{k}\left(z^{2 M}\right)$. For simplicity, we assume that $M$ is even. Similar conditions can be derived when $M$ is an odd number. $n_{k}$ 's are usually chosen to be identical for all $k$, and it determines the delay of the filter bank. In our IIR CMFB, $H_{k}(z)$ 's are assumed to have the same denominator (i.e., $H_{k}(z)=N_{k}(z) / D(z)$ for $\left.k=0,1, \cdots, 2 M-1\right)$. The PR condition in (3) is then simplified to

$$
\begin{aligned}
& N_{k}(z) N_{2 M-k-1}(z)+N_{M+k}(z) N_{M-k-1}(z) \\
& \quad=\beta \cdot z^{-n_{k}} D^{2}(z), \quad k=0,1, \cdots,(M / 2)-1 .
\end{aligned}
$$

To ensure that the analysis and synthesis filters be causal stable, all the roots of $D(z)$ shall remain inside the unit circle. For simplicity, all zeros of $D(z)$ are assumed to occur in complex 
conjugate pairs so that $D(z)=K_{0} \prod_{k=1}^{N D_{i}}\left(1-p_{i, k} \cdot z^{-1}\right)(1-$ $\left.p_{i, k}^{*} \cdot z^{-1}\right)$, where $K_{0}$ is a constant. Modifications to include a fixed number of real zeros are easily made.

\section{Design Procedure AND Design ExAMPLES}

Due to the cosine modulation in (2), the analysis filter $f_{k}(n)$ is obtained by shifting $H(z)$ to $\omega= \pm(k+1 / 2) \pi / M$. For $k=1, \cdots, M-2$, these two copies will be widely separated, and the analysis filters look very like the original prototype filter. However, for $k=0$ and $M-1$, the two copies will overlap each other in the transition bands. In the case of orthogonal CMFB, the linear-phase property of $H(z)$ ensures that the passband of $f_{0}(n)$ and $f_{M-1}(n)$ will be flat. However, in biorthogonal CMFB, $H(z)$ is no longer symmetric, and there will be bumping in the passband if the stopband attenuation of $H(z)$ alone is minimized. These bumps have been observed in the design examples of other literatures [3], [4], [7]. This can be suppressed by minimizing the passband ripples of $F_{0}\left(e^{j \omega}\right)$ $\left(F_{M-1}\left(e^{j \omega}\right)\right)$ around $\omega=0(\omega=\pi)$, at the expense of decreased stopband attenuation. As current IIR CMFB is biorthogonal, the following objective function is used:

$$
\begin{gathered}
\Phi_{x}=\alpha \cdot \int_{\omega_{s}}^{\pi}\left|H\left(e^{j \omega}\right)\right|^{2} d \omega+(1-\alpha) \cdot \int_{0}^{\omega_{p}-\varepsilon} \\
\cdot\left(A-\left|F_{0}\left(e^{j \omega}\right)\right|\right)^{2} d \omega, \quad \varepsilon>0
\end{gathered}
$$

where

$\omega_{s} \quad$ stopband cutoff frequency whose value should be between $\pi /(2 M)$ and $\pi / M$;

$\omega_{p} \quad$ passband cutoff frequency of $F_{0}\left(e^{j \omega}\right)$;

$\varepsilon \quad$ constant less than $\pi /(2 M)$ used to suppress the bumping around $\omega=0$;

$\alpha \quad$ constant between 0 and 1 used to control the relative weighting of the stopband attenuation and the suppression of the bump;

$A \quad$ desired response of $F_{0}\left(e^{j \omega}\right)$ at $\omega=0$;

$X \quad$ vector containing the coefficients of $N_{k}(z)$ and the real and imaginary parts of $p_{i, k}$ 's.

The design problem can be formulated as the following constrained nonlinear optimization problem:

$\min \Phi_{X}, \quad$ subjected to the PR condition in (4) and

$$
\left|p_{i, k}\right|<1, i=0,1, k=1, \cdots, N D_{i} .
$$

This constrained nonlinear optimization can be solved by the NCONF/DCONF subroutine in the IMSL library, which is based on sequential quadratic programming with an augmented Lagrangian line-search method. The initial guess to the optimization program is obtained by designing an $M$-channel, biorthogonal PR FIR CMFB with the desired delay. The initial values $p_{i, k}$ are chosen to be zero. The magnitude response of the prototype filter and analysis filters of a four-channel IIR CMFB designed by the proposed method are shown in Figs. 2 and 3(a). The order of $N_{k}(z)(k=0,1, \cdots, 2 M-1)$ and $D(z)$ are, respectively, 4 and 2 . The magnitude responses of the numerator and denominator of the prototype filter are also shown in dotted and dashed lines, respectively. In this design example, the DCONF program converged after about 150

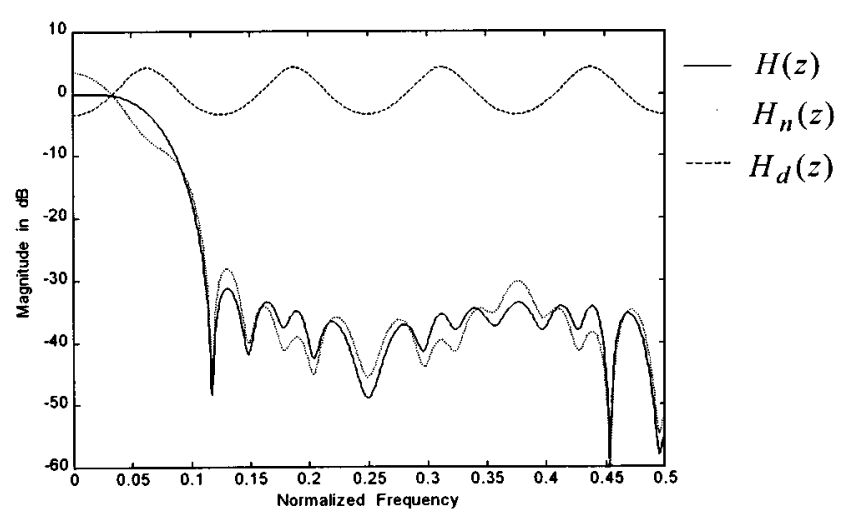

Fig. 2. Magnitude responses of four-channel IIR CMFB: prototype filter $H(z)$ (solid line), numerator $H_{n}(z)$ (dotted line), and denominator $H_{d}(z)$ (dashed line).

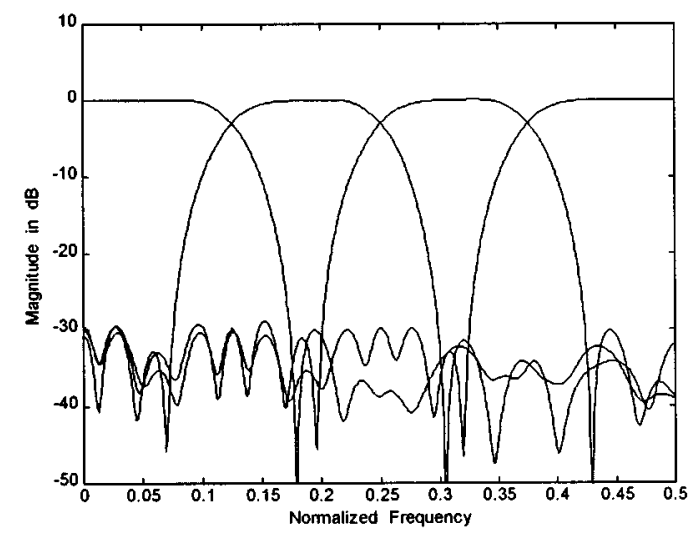

(a)

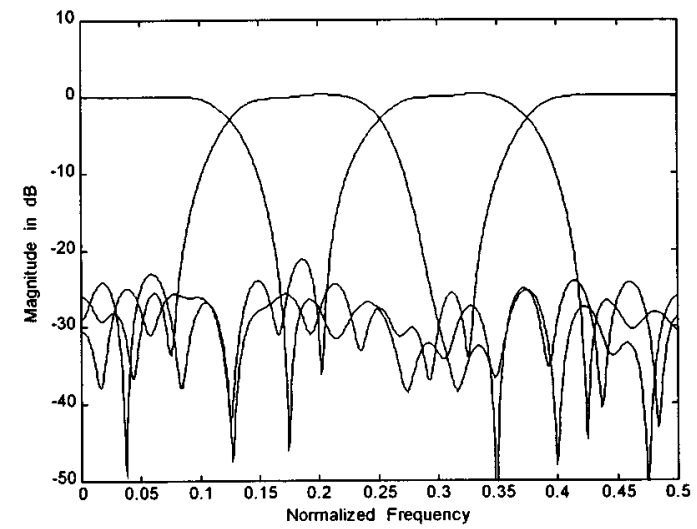

(b)

Fig. 3. Magnitude responses of four-channel biorthogonal CMFB: (a) IIR analysis filters and (b) FIR analysis filters.

iterations. Due to roundoff errors and the inherent limitation of the optimization program, the PR constraints can only be met approximately. In this particular design example, the violation of PR constraints is of the order of $10^{16}$. In general, the number of iterations used and the amount of constraint violation will depend on the difficulty of the problem and the initial guess used. To obtain a PR solution, the factorization technique recently developed in [9] is used to convert the nearly PR solution to a structurally PR system with a slight decrease in stopband attenuation. Due to page limitation, details of this factorization are omitted here, and interested readers are referred to [9] for the derivations. Table I lists the polyphase components $N_{k}(z)$ and 
TABLE I

POLYPHASE COMPONENTS OF IIR CMFB IN EXAMPLE $1 \beta=3.130696027842296 e-002, \eta_{k}=2$, $k=0,1, \cdots,(M / 2)-1$

\begin{tabular}{|c|c|c|}
\hline $\mathrm{n}$ & $N_{0}(z)$ & $N_{7}(z)$ \\
\hline 0 & $-3.125579445445457 \mathrm{e}-003$ & $5.131102949572527 \mathrm{e}-002$ \\
\hline 1 & $7.835422946454861 \mathrm{e}-002$ & $1.036074866008372 \mathrm{e}-001$ \\
\hline 2 & $8.420258281000559 \mathrm{e}-002$ & $3.377592682063479 \mathrm{e}-002$ \\
\hline 3 & $2.540743531696396 \mathrm{e}-002$ & $2.837070581719559 \mathrm{e}-003$ \\
\hline 4 & $2.382617025834014 \mathrm{e}-003$ & $-1.837322324691471 \mathrm{e}-004$ \\
\hline $\mathrm{n}$ & $N_{1}(z)$ & $N_{6}(z)$ \\
\hline 0 & $-1.600091322669139 \mathrm{e}-003$ & $2.698192299073904 \mathrm{e}-002$ \\
\hline 1 & $1.087163944344360 \mathrm{e}-001$ & $1.235497579354557 \mathrm{e}-001$ \\
\hline 2 & $6.857102162062063 \mathrm{e}-002$ & $4.153601572557963 \mathrm{e}-002$ \\
\hline 3 & $1.606833288080864 \mathrm{e}-002$ & $1.313837619211361 \mathrm{e}-003$ \\
\hline 4 & $3.706573603708072 \mathrm{e}-003$ & $-1.729779976911428 \mathrm{e}-004$ \\
\hline $\mathbf{n}$ & $N_{2}(z)$ & $N_{5}(z)$ \\
\hline 0 & $-1.831376700806724 \mathrm{e}-002$ & $-2.357436393473307 \mathrm{e}-003$ \\
\hline 1 & $1.279333689371270 \mathrm{e}-001$ & $1.329104878273331 \mathrm{e}-001$ \\
\hline 2 & $6.017889555528450 \mathrm{e}-002$ & $4.206994115288004 \mathrm{e}-002$ \\
\hline 3 & $8.015289850629751 \mathrm{e}-003$ & $-6.510818843298294 \mathrm{e}-004$ \\
\hline 4 & $-1.630448510605056 \mathrm{e}-003$ & $-3.932388395548742 \mathrm{e}-004$ \\
\hline $\mathrm{n}$ & $N_{3}(z)$ & $N_{4}(z)$ \\
\hline 0 & $-1.580682191601748 \mathrm{e}-002$ & $-1.014604326970816 \mathrm{e}-002$ \\
\hline 1 & $1.417235349573783 \mathrm{e}-001$ & $1.428920780308221 \mathrm{e}-001$ \\
\hline 2 & $5.188330124351559 \mathrm{e}-002$ & $4.750079231828455 \mathrm{e}-002$ \\
\hline 3 & $2.523146053594161 \mathrm{e}-003$ & $-4.557082964174990 \mathrm{e}-004$ \\
\hline 4 & $-4.782722743037482 \mathrm{e}-004$ & $-9.153019499318281 \mathrm{e}-004$ \\
\hline $\mathrm{n}$ & \multicolumn{2}{|c|}{$D(z)$} \\
\hline 0 & \multicolumn{2}{|c|}{$1.000000000000000 \mathrm{e}+000$} \\
\hline 1 & \multicolumn{2}{|c|}{$4.279018931760565 \mathrm{e}-001$} \\
\hline 2 & \multicolumn{2}{|c|}{$4.582067643614702 \mathrm{e}-002$} \\
\hline
\end{tabular}

the denominator $D(z)$ of this PR IIR CMFB. For comparison, the magnitude response of a four-channel biorthogonal FIR CMFB with similar complexity (40-tap) is shown in Fig. 3(b). Both of them have the same system delay of 23 samples. It can be seen that the poles help to sharpen the transition band and increase the stopband attenuation of the CMFB (about 7 $\mathrm{dB}$ better than the FIR CMFB). The passband flatness of the proposed IIR CMFB is also significantly improved. Fig. 4 shows the magnitude responses of another four-channel IIR CMFB designed using the proposed method. The orders of the numerator and denominator of the prototype filter are

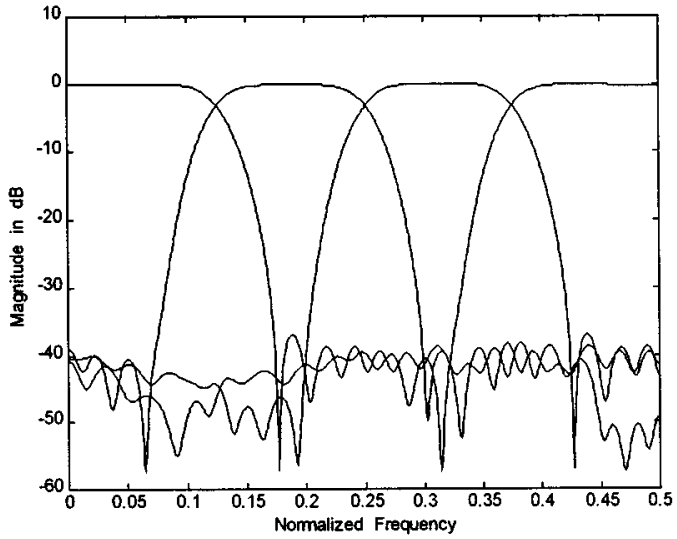

Fig. 4. Magnitude responses of four-channel, IIR, cosine-modulated analysis filters. System delay $=37$.

45 and 16, respectively. The system delay is increased to 37 samples. It can be seen that CMFB with larger system delay has higher stopband attenuation (40 versus 30 ) than its low-delay counterparts. It is also observed that the bump of the low-delay FIR CMFB at $\omega=0$ and $\pi$ is very difficult to suppress without significantly decreasing its stopband attenuation.

\section{CONCLUSION}

A new method for designing a class of $M$-channel causalstable, PR IIR CMFB is presented. The proposed CMFB has the same denominator for all its polyphase components in the prototype filter. Therefore, the PR condition is considerably simplified, and it is relatively simple to satisfy the PR and the casual-stable requirements of the IIR CMFB. Design examples show that proposed IIR CMFB has sharper cutoff and higher stopband attenuation and passband flatness than its FIR counterparts, especially when the system delay is small.

\section{REFERENCES}

[1] P. P. Vaidyanathan, Multirate Systems and Filter Banks. Englewood Cliffs, NJ: Prentice-Hall, 1992.

[2] R. D. Koilpillai and P. P. Vaidyanathan, "Cosine-modulated FIR filter banks satisfying perfect reconstruction," IEEE Trans. Signal Processing, vol. 40, pp. 770-783, Apr. 1992.

[3] T. Q. Nguyen and P. N. Heller, "Biorthogonal cosine-modulated filter banks," in IEEE Proc. ICASSP'96, vol. 3, May 1996, pp. 1471-1474.

[4] S. C. Chan, L. Yi, and K. L. Ho, " $M$-channel compactly supported biorthogonal cosine-modulated wavelets bases," IEEE Trans. Signal Processing: Special Issue Theory Applicat. Filter Banks Wavelets, vol. 46, pp. 1142-1151, Apr. 1998.

[5] T. Q. Nguyen, T. I. Laakso, and T. E. Tuncer, "On perfect-reconstruction allpass-based cosine-modulated IIR filter banks," in Proc. IEEE ISCAS-94, vol. 2, May 1994, pp. 33-36.

[6] S. Znidar, "Cosine-modulated perfect reconstruction IIR filter banks with good time-frequency resolution," in Proc. 13th Int. Conf. Digital Signal Processing, vol. 2, July 1997, pp. 1067-1070.

[7] J. S. Mao, S. C. Chan, and K. L. Ho, "Theory and design of causal stable IIR PR cosine-modulated filter banks," in Proc. IEEE ISCAS'99, vol. 3, June 1999, pp. 427-430.

[8] H. S. Malvar, "Extended lapped transforms: Properties, applications, and fast algorithms," IEEE Trans. Signal Processing, vol. 40, pp. 2703-2714, Nov. 1992.

[9] S. C. Chan, "Factorization of $M$-channel biorthogonal cosine-modulated filter banks," Dept. Elect. Electron. Eng., Univ. Hong Kong, Kowloon, internal rep., Dec. 1998. 